SUPPORTING INFORMATION FOR:

\title{
Supersonic Jet Spectroscopy and DFT Study of Isomeric Diazines: 1,4- and 1,8-Diazatriphenylene. Why Do They Differ so Deeply?
}

\author{
Michał Kijak, Sebastian Peukert, Ephriem Mengesha, Jerzy Sepioł, and Michał Gil* \\ Institute of Physical Chemistry, Polish Academy of Sciences, Kasprzaka 44/52, PL-01-224 \\ Warsaw, Poland; (*Corresponding Author: mgil@ichf.edu.pl)
}

\section{Synthesis of 1,4-DAT:}

1,4-diazatriphenylene (phenanthro[9,10-b]pyrazine) was synthesized as follows: $3.6 \mathrm{~g}$ $(0.06 \mathrm{~mol})$ of ethylenediamine was added to the mixture of $10.4 \mathrm{~g}(0.05 \mathrm{~mol})$ of 9,10 phenanthrenequinone in $200 \mathrm{ml}$ of toluene. The suspension was heated under reflux for $24 \mathrm{~h}$ using Dean-Stark apparatus. The resulting suspension was treated with $10 \mathrm{~g}$ of manganese(IV) oxide and heated continuously under reflux until the intermediate product disappears on the TLC. The hot black suspension was filtered and the solid residue rinsed with toluene. The solution was concentrated and resulting crude product were purified by column chromatography, giving $9.9 \mathrm{~g}(86 \%)$ of $1,4-\mathrm{DAT}$. 
A)
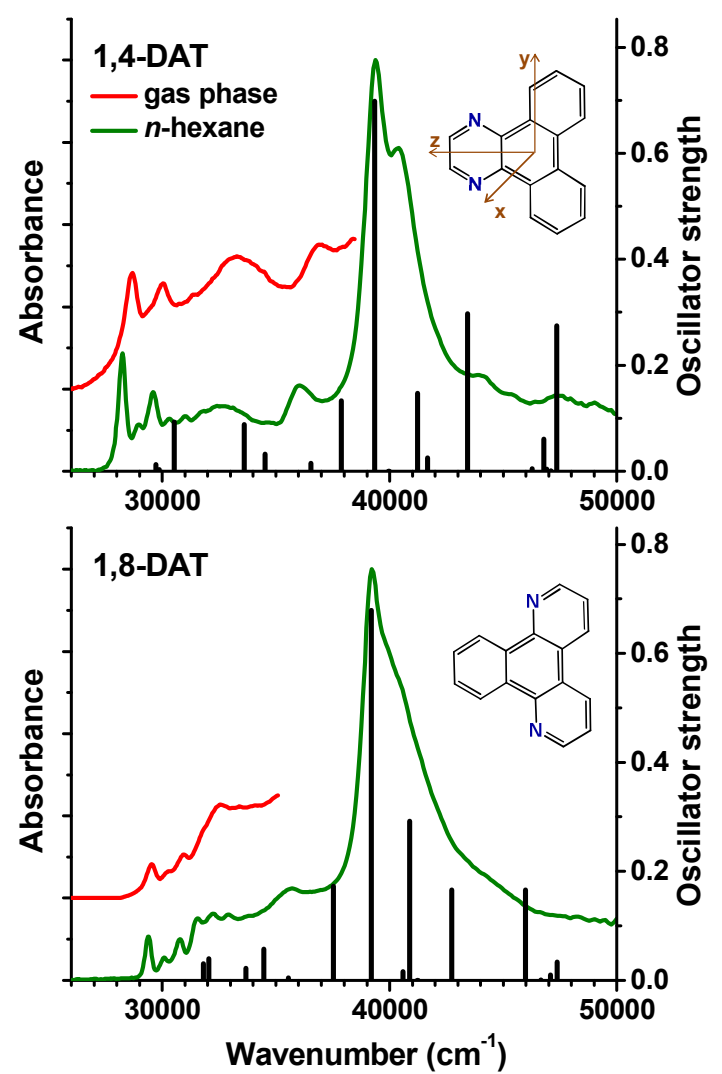

B)
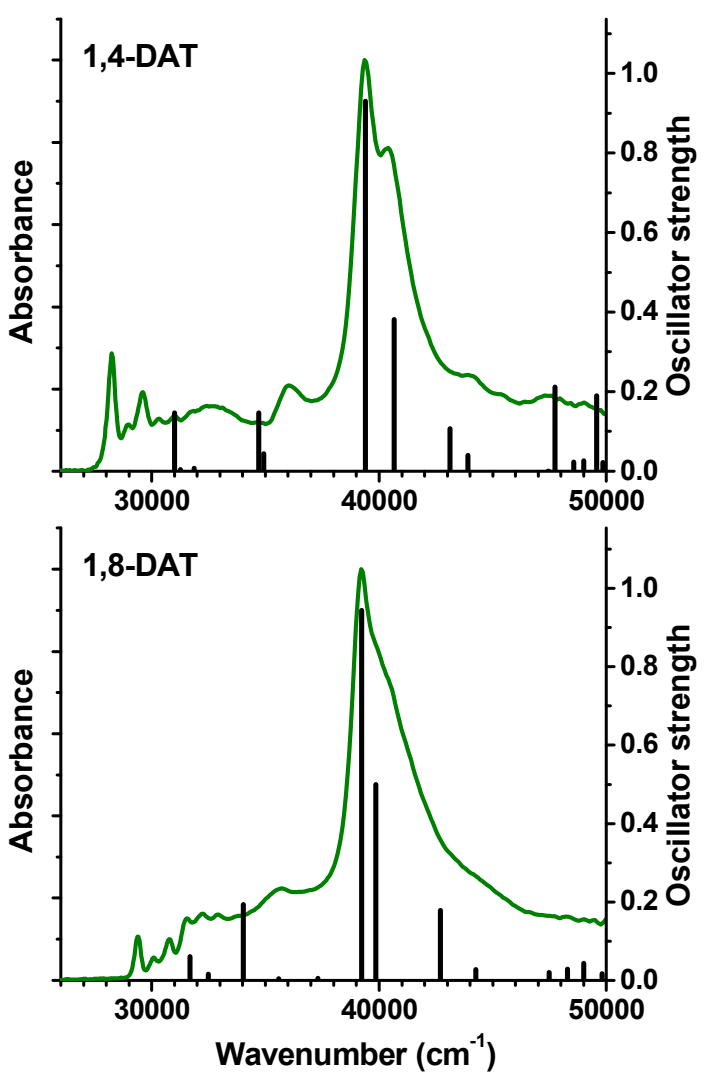

Figure S1. Normalized absorption spectra of 1,4-DAT and 1,8-DAT in $n$-hexane solution. Bars show the energy and the normalized oscillator strength of the lowest twenty electronic transitions calculated at the TD-DFT level using (A) B3LYP functional and, (B) BHLYP functional (shifted by -3010 and $-3600 \mathrm{~cm}^{-1}$, for $1,4-\mathrm{DAT}$ and 1,8 -DAT, respectively, to match the position of the strongest absorption band) at the DFT/B3LYP optimized ground state geometries. The gas phase absorption spectra $\left(\right.$ at $\left.100^{\circ} \mathrm{C}\right)$ are shown in the left side panels. 


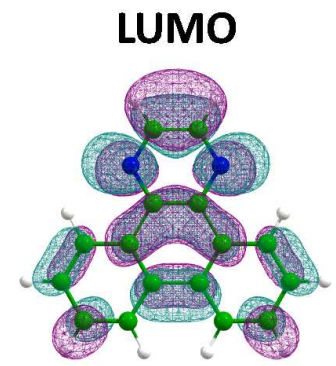

HOMO

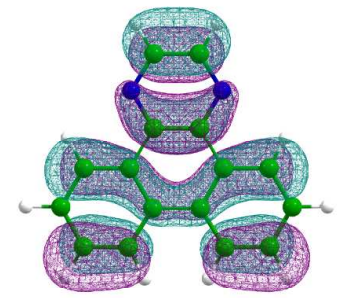

HOMO-1

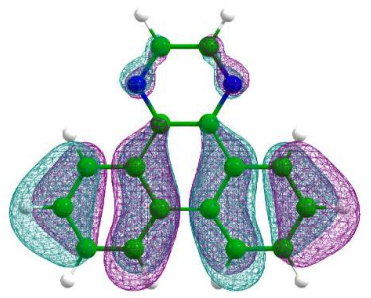

HOMO-3

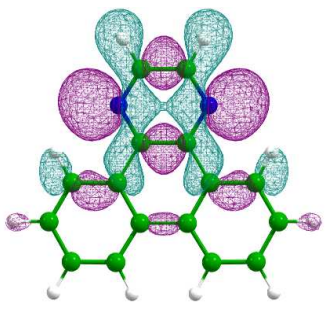

LUMO+1

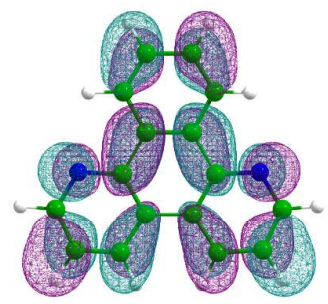

LUMO

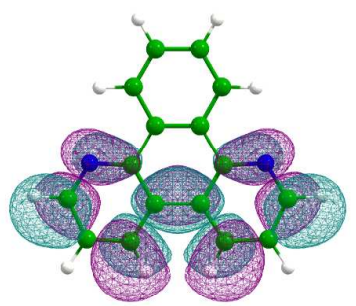

HOMO

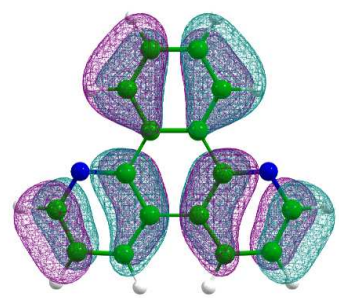

HOMO-4

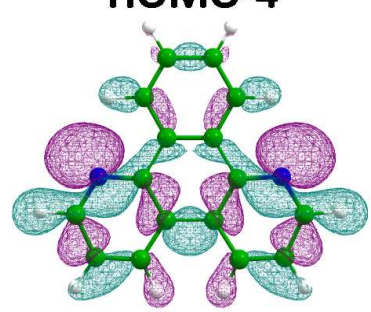

\begin{tabular}{l|l|ccc|c|lccc}
\hline \multicolumn{5}{c|}{ 1,4-DAT } & \multicolumn{5}{c}{ 1,8-DAT } \\
\hline & Symmetry & $E / \mathrm{cm}^{-1}$ & Config. & $\mu / \mathrm{D}$ & & Symmetry & $E / \mathrm{cm}^{-1}$ & Config. & $\mu / \mathrm{D}$ \\
\hline $\mathrm{S}_{0}$ & $\mathrm{~A}_{1}$ & 0 & - & 0.7 & $\mathrm{~S}_{0}$ & $\mathrm{~A}_{1}$ & 0 & - & 3.0 \\
$\mathrm{~S}_{1}$ & $\mathrm{~A}_{1}\left(\pi, \pi^{*}\right)$ & 34020 & $\mathrm{H}-\mathrm{L}$ & 3.2 & $\mathrm{~S}_{1}$ & $\mathrm{~A}_{1}\left(\pi, \pi^{*}\right)$ & 35290 & $\mathrm{H}-\mathrm{L}+1$ & 1.8 \\
$\mathrm{~S}_{2}$ & $\mathrm{~B}_{1}\left(\mathrm{n}, \pi^{*}\right)$ & 34270 & $\mathrm{H}-3-\mathrm{L}$ & -0.1 & $\mathrm{~S}_{2}$ & $\mathrm{~B}_{2}\left(\pi, \pi^{*}\right)^{\mathrm{CT}}$ & 36090 & $\mathrm{H}-\mathrm{L}$ & 0.7 \\
$\mathrm{~S}_{3}$ & $\mathrm{~B}_{2}\left(\pi, \pi^{*}\right)^{\mathrm{CT}}$ & 34880 & $\mathrm{H}-1-\mathrm{L}$ & 5.1 & $\mathrm{~S}_{5}$ & $\mathrm{~B}_{1}\left(\mathrm{n}, \pi^{*}\right)$ & 40900 & $\mathrm{H}-4-\mathrm{L}$ & 1.9 \\
\hline
\end{tabular}

Figure S2. Contour plots of main molecular orbitals involved in the low-energy electronic transitions of 1,4-DAT (left) and 1,8-DAT (right) calculated at the TD-DFT/BHLYP level for the DFT/B3LYP optimized ground state geometries. Relevant electronic states are listed in the table (bottom) with their relative energies $(E)$, dominant electronic configurations and dipole moments ( $\mu$, minus sign refers to a reversed direction). 

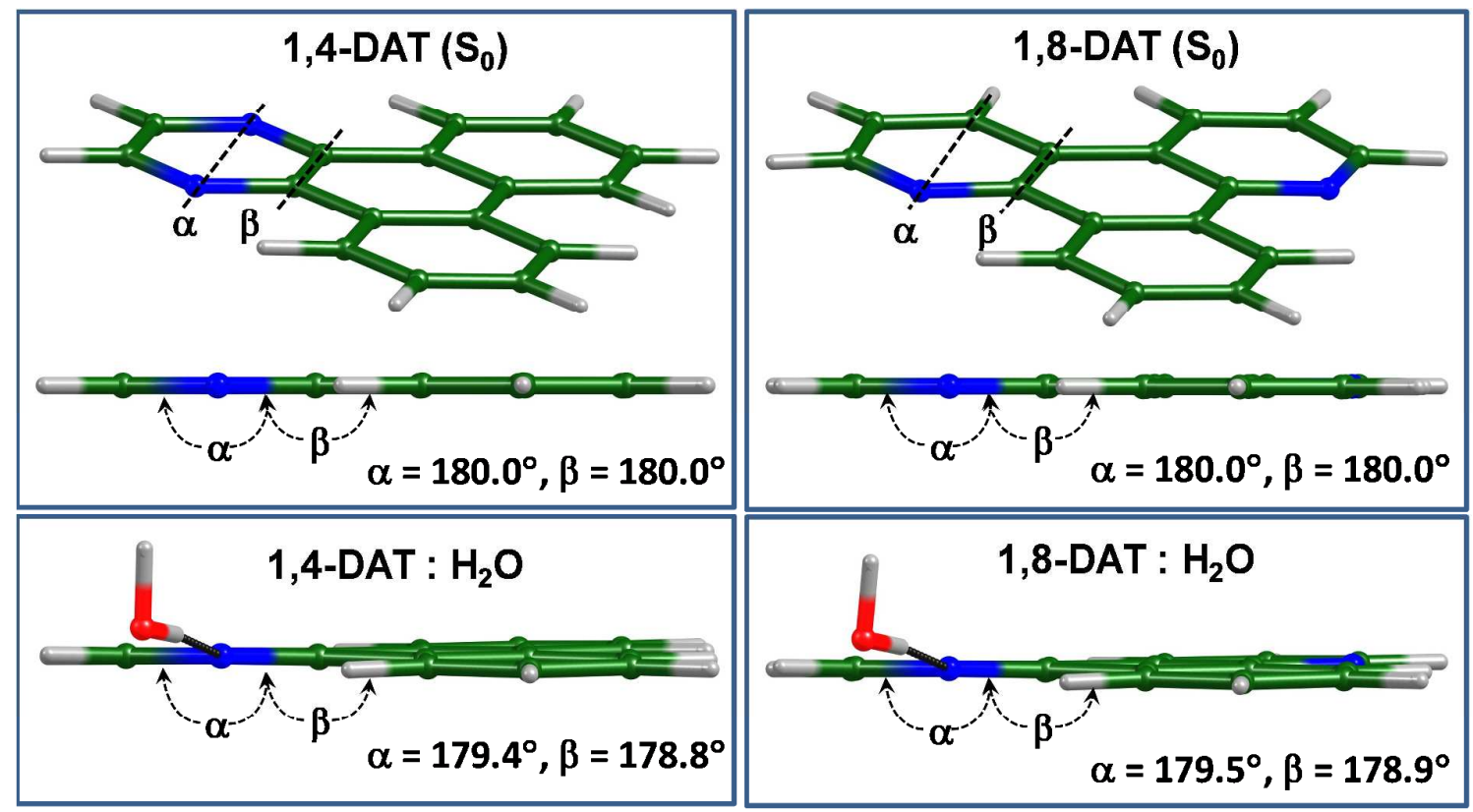

Figure S3. The ground-state geometries of 1,4- and 1,8-DAT and their 1:1 complex with water calculated using DFT/B3LYP approach. Values of dihedral angles $\alpha$ and $\beta$ (see text for description) are listed. 


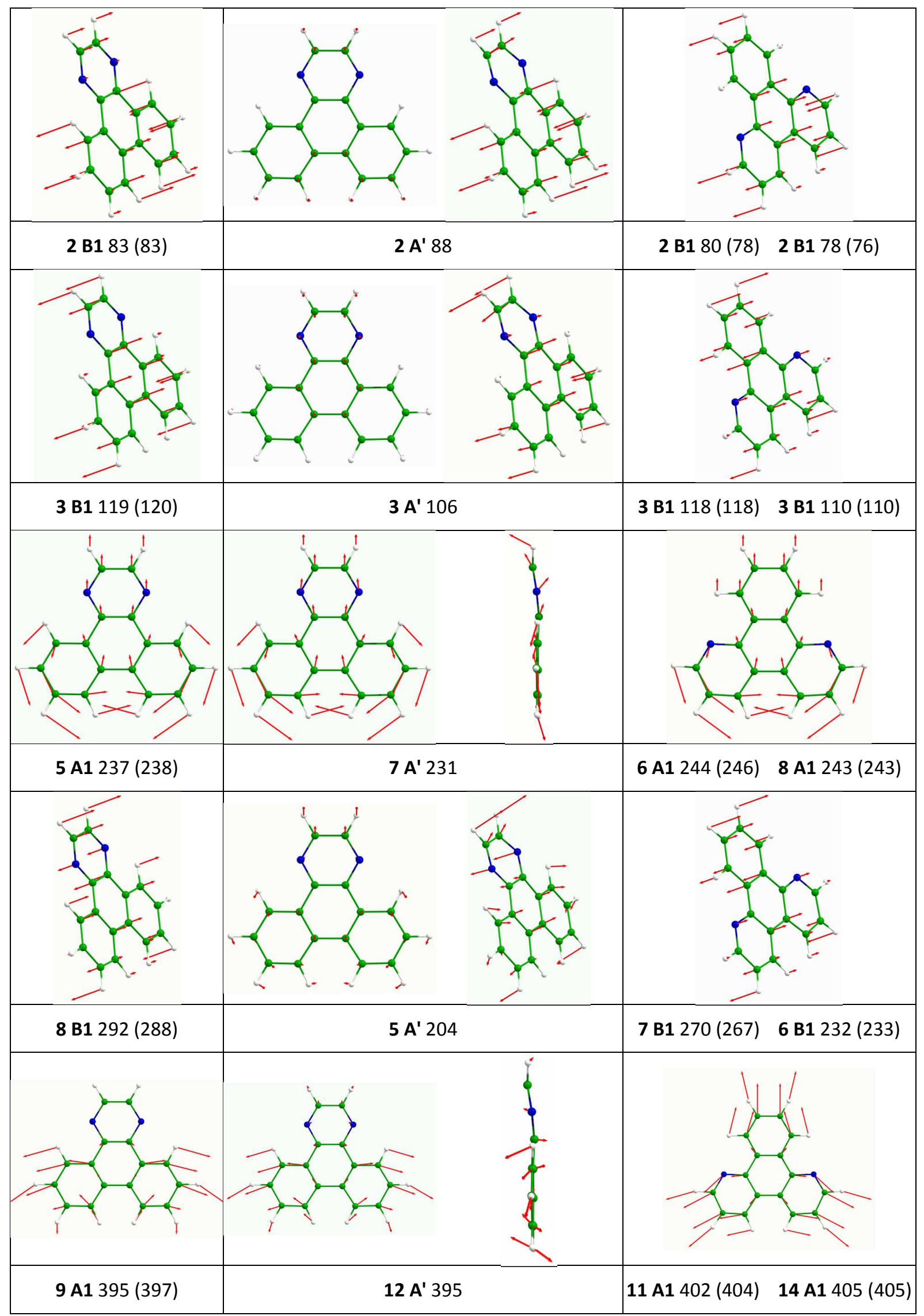




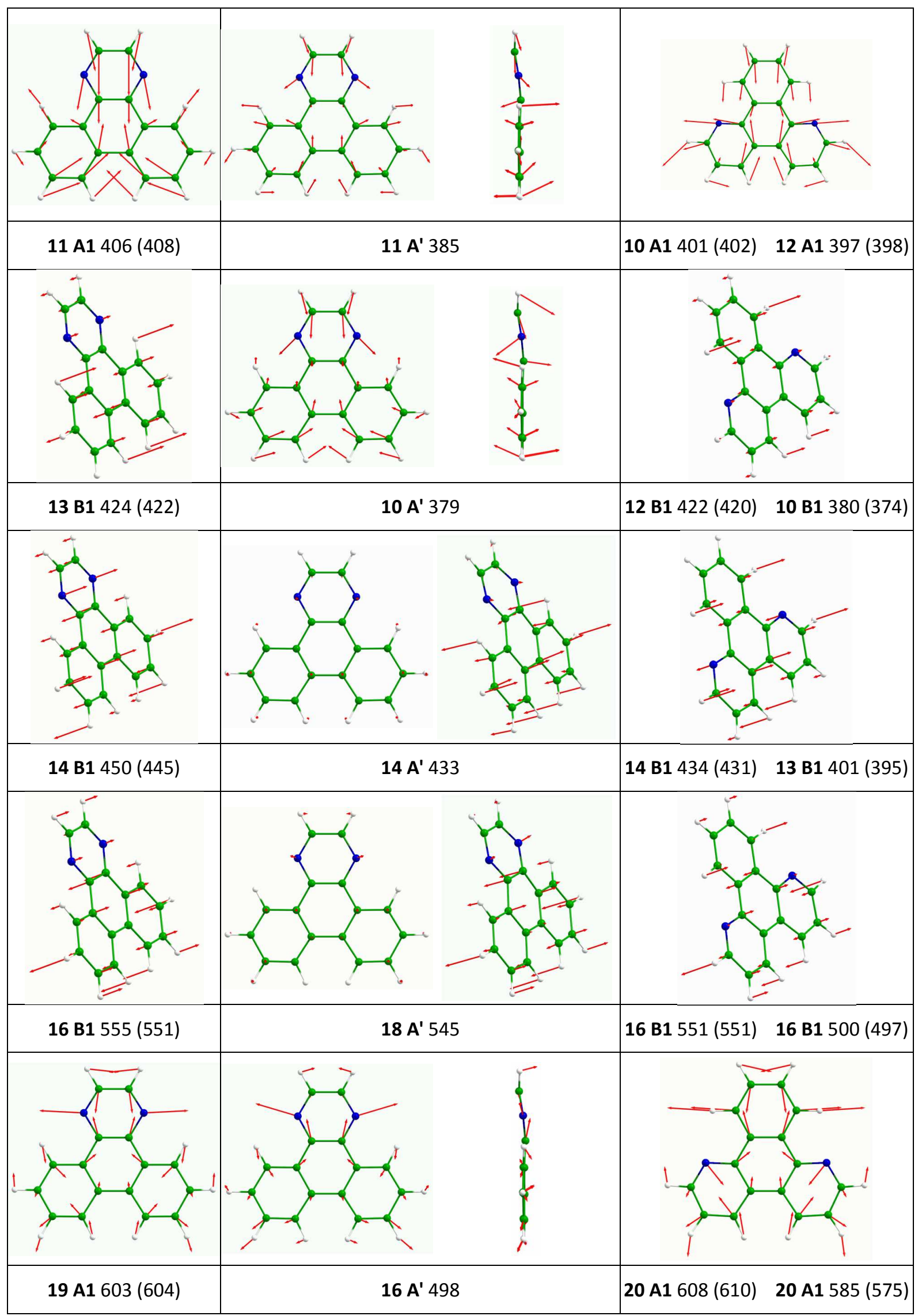




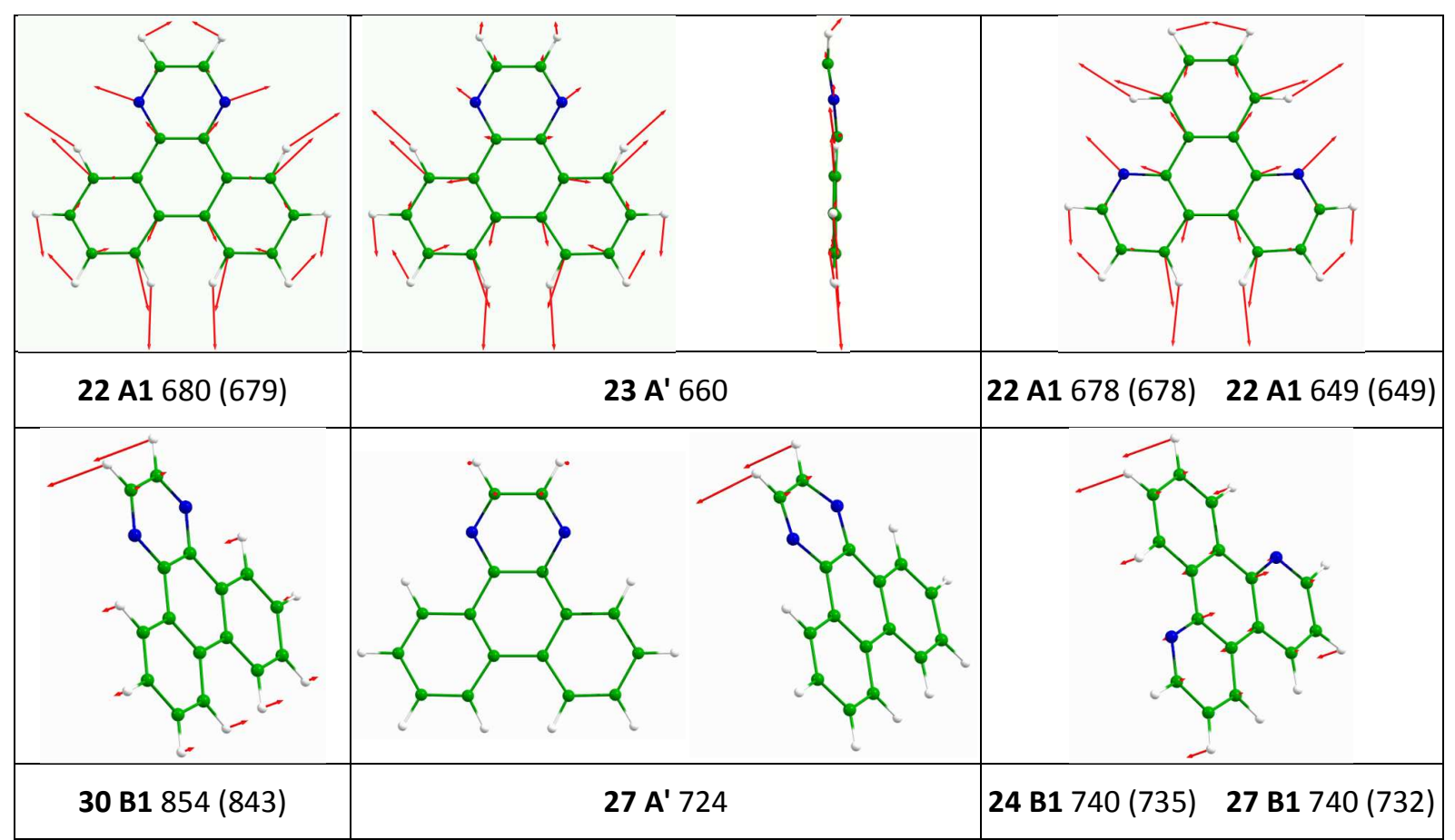

Figure S4. Shapes of the $\mathrm{A}^{\prime}$ type (in $C_{\mathrm{s}}$ symmetry, A1 and B1 in $C_{2 \mathrm{v}}$ symmetry) vibrational modes with energies below $700 \mathrm{~cm}^{-1}$ (plus that of the characteristic $\mathrm{C}-\mathrm{H}$ oop mode) of 1,4DAT in the $\mathrm{S}_{0}\left(1^{\text {st }}\right.$ column $)$ and $\mathrm{S}_{1}\left(2^{\text {nd }}\right.$ column $)$ states and 1,8 -DAT in the $\mathrm{S}_{1}$ state $\left(3^{\text {rd }}\right.$ column), calculated at the (TD)DFT/BHLYP/6-31+G(d,p) level. Mode number, symmetry, and energy in $\mathrm{cm}^{-1}$ (values obtained with B3LYP functional, in brackets) are given. For 1,8DAT the $\mathrm{S}_{0}$ and $\mathrm{S}_{1}$ states values are listed consecutively in the last column. 


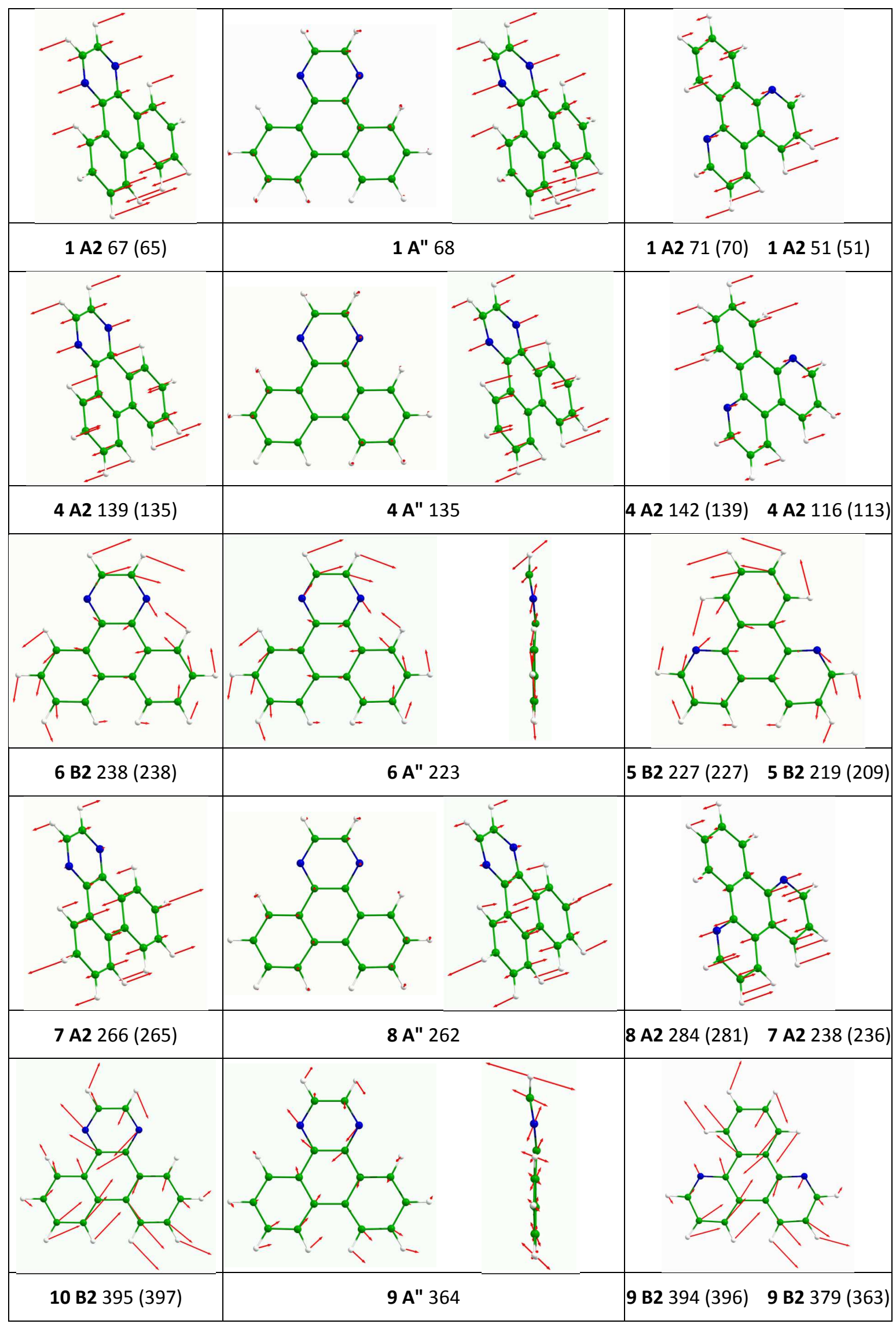


Figure S4-A. Shapes of the A" type (in $C_{\mathrm{s}}$ symmetry, A2 and B2 in $C_{2 \mathrm{v}}$ symmetry) vibrational modes with energies below $400 \mathrm{~cm}^{-1}$. For details, see description of Figure S4.

\begin{tabular}{|r|c|l|}
\hline $\begin{array}{c}\Delta \mathrm{E} \\
\left(\mathrm{cm}^{-1}\right)\end{array}$ & $\begin{array}{c}\mathrm{FC} \\
\text { factor }\end{array}$ & Assignment \\
\hline 0.0 & 0.2976 & $\mid 0^{\wedge}$ \\
101.6 & 0.0051 & $\mid 1^{\wedge} 2>$ \\
166.6 & 0.0014 & $\left|4^{\wedge} 1 ; 1^{\wedge} 1\right\rangle$ \\
231.6 & 0.0011 & $\mid 4^{\wedge} 2>$ \\
289.3 & 0.0007 & $\left|7^{\wedge} 1 ; 1^{\wedge} 1\right\rangle$ \\
309.6 & 0.0005 & $\left|6^{\wedge} 1 ; 2^{\wedge} 1\right\rangle$ \\
397.0 & 0.0025 & $\left|1^{\wedge} 1\right\rangle$ \\
405.2 & 0.0046 & $\mid 1^{\wedge} 1>$ \\
464.1 & 0.0008 & $\mid 6^{\wedge} 2>$ \\
477.0 & 0.0010 & $\mid 7^{\wedge} 2>$ \\
584.7 & 0.0118 & $\mid 2^{\wedge} 1>$ \\
648.7 & 0.0644 & $\mid 2^{\wedge} 1>$ \\
\hline
\end{tabular}

\begin{tabular}{|c|c|c|}
\hline $\begin{array}{c}\Delta \mathrm{E} \\
\left(\mathrm{cm}^{-1}\right)\end{array}$ & $\begin{array}{c}\text { FC } \\
\text { factor }\end{array}$ & Assignment \\
\hline 0.0 & 0.2976 & $\mid 0>$ \\
\hline-141.7 & 0.0028 & $\mid 1^{\wedge} 2>$ \\
\hline-400.6 & 0.0043 & $\left|10^{\wedge} 1\right\rangle$ \\
\hline-608.2 & 0.0146 & $\mid 20^{\wedge} 1>$ \\
\hline-678.3 & 0.0407 & $\left|22^{\wedge} 1\right\rangle$ \\
\hline-999.3 & 0.0040 & $\mid 40^{\wedge} 1>$ \\
\hline-1029.0 & 0.0070 & $\left|41^{\wedge} 1\right\rangle$ \\
\hline-1047.6 & 0.0109 & $\mid 42^{\wedge} 1>$ \\
\hline-1131.5 & 0.0039 & $\mid 47^{\wedge} 1>$ \\
\hline-1173.0 & 0.0095 & $\mid 48^{\wedge} 1>$ \\
\hline-1213.0 & 0.0068 & $\mid 50^{\wedge} 1>$ \\
\hline-1307.5 & 0.0368 & $\mid 54^{\wedge} 1>$ \\
\hline-1321.9 & 0.0365 & $\mid 56^{\wedge} 1>$ \\
\hline-1356.5 & 0.0036 & $\mid 22^{\wedge} 2>$ \\
\hline-1385.5 & 0.0947 & $\mid 57^{\wedge} 1>$ \\
\hline-1433.8 & 0.0487 & $\mid 59^{\wedge} 1>$ \\
\hline-1483.1 & 0.0026 & $\mid 62^{\wedge} 1>$ \\
\hline-1530.8 & 0.0063 & $\mid 63^{\wedge} 1>$ \\
\hline-1985.8 & 0.0060 & $\mid 54^{\wedge} 1 ; 22^{\wedge} 1>$ \\
\hline-1993.7 & 0.0050 & $\mid 57^{\wedge} 1 ; 20^{\wedge} 1>$ \\
\hline-2000.2 & 0.0053 & $\mid 56^{\wedge} 1 ; 22^{\wedge} 1>$ \\
\hline-2063.8 & 0.0148 & $\mid 57^{\wedge} 1 ; 22^{\wedge} 1>$ \\
\hline-2112.1 & 0.0081 & $\mid 59^{\wedge} 1 ; 22^{\wedge} 1>$ \\
\hline
\end{tabular}

Table S1. List of most intense (FC factor larger than 0.00025 and 0.0025 , for absorption and emission, respectively) vibronic bands in analyzed region of energies in the (TD)DFT/BHLYP simulated absorption (left) and emission (right) spectra of 1,8-DAT. 


\begin{tabular}{|r|l|l|}
\hline $\begin{array}{r}\Delta \mathrm{E} \\
\left(\mathrm{cm}^{-1}\right)\end{array}$ & $\begin{array}{c}\mathrm{FC} \\
\text { factor }\end{array}$ & ||$^{\text {Assignment }}$ \\
\hline 0.0 & 0.0258 & $\mid 0^{\wedge}$ \\
88.3 & 0.0009 & $\mid 2^{\wedge} 1>$ \\
105.9 & 0.0322 & $\mid 3^{\wedge} 1>$ \\
194.2 & 0.0009 & $\mid 3^{\wedge} 1 ; 2^{\wedge} 1>$ \\
203.6 & 0.0064 & $\mid 5^{\wedge} 1>$ \\
211.8 & 0.0173 & $\mid 3^{\wedge} 2>$ \\
300.0 & 0.0004 & $\mid 3^{\wedge} 2 ; 2^{\wedge} 1>$ \\
309.5 & 0.0054 & $\mid 5^{\wedge} 1 ; 3^{\wedge} 1>$ \\
317.6 & 0.0051 & $\mid 3^{\wedge} 3>$ \\
379.0 & 0.0003 & $\mid 10^{\wedge} 1>$ \\
384.6 & 0.0094 & $\mid 11^{\wedge} 1>$ \\
415.4 & 0.0016 & $\mid 5^{\wedge} 1 ; 3^{\wedge} 2>$ \\
423.5 & 0.0009 & $\mid 3^{\wedge} 4>$ \\
433.3 & 0.0014 & $\mid 14^{\wedge} 1>$ \\
472.9 & 0.0006 & $\mid 11^{\wedge} 1 ; 2^{\wedge} 1>$ \\
484.9 & 0.0005 & $\mid 10^{\wedge} 1 ; 3^{\wedge} 1>$ \\
490.5 & 0.0134 & $\mid 11^{\wedge} 1 ; 3^{\wedge} 1>$ \\
498.3 & 0.0006 & $\mid 16^{\wedge} 1>$ \\
539.2 & 0.0019 & $\mid 14^{\wedge} 1 ; 3^{\wedge} 1>$ \\
544.6 & 0.0070 & $\mid 18^{\wedge} 1>$ \\
578.8 & 0.0007 & $\mid 11^{\wedge} 1 ; 3^{\wedge} 1 ; 2^{\wedge} 1>$ \\
588.2 & 0.0030 & $\mid 11^{\wedge} 1 ; 5^{\wedge} 1>$ \\
590.7 & 0.0003 & $\mid 10^{\wedge} 1 ; 3^{\wedge} 2>$ \\
596.3 & 0.0083 & $\mid 11^{\wedge} 1 ; 3^{\wedge} 2>$ \\
604.2 & 0.0008 & $\mid 16^{\wedge} 1 ; 3^{\wedge} 1>$ \\
632.9 & 0.0003 & $\mid 18^{\wedge} 1 ; 2^{\wedge} 1>$ \\
636.9 & 0.0004 & $\mid 14^{\wedge} 1 ; 5^{\wedge} 1>$ \\
645.0 & 0.0012 & $\mid 14^{\wedge} 1 ; 3^{\wedge} 2>$ \\
650.5 & 0.0090 & $\mid 18^{\wedge} 1 ; 3^{\wedge} 1>$ \\
660.5 & 0.0005 & $\mid 23^{\wedge} 1>$ \\
684.6 & 0.0004 & $\mid 11^{\wedge} 1 ; 3^{\wedge} 2 ; 2^{\wedge} 1>$ \\
693.4 & 0.0012 & $\mid 25^{\wedge} 1>$ \\
694.1 & 0.0031 & $\mid 11^{\wedge} 1 ; 5^{\wedge} 1 ; 3^{\wedge} 1>$ \\
\hline
\end{tabular}

\begin{tabular}{|c|c|c|}
\hline $\begin{array}{c}\Delta \mathrm{E} \\
\left(\mathrm{cm}^{-1}\right)\end{array}$ & $\begin{array}{c}\text { FC } \\
\text { factor }\end{array}$ & Assignment \\
\hline 0.0 & 0.0258 & $\mid 0>$ \\
\hline-118.7 & 0.0283 & $\mid 3^{\wedge} 1>$ \\
\hline-237.4 & 0.0164 & $\mid 3^{\wedge} 2>$ \\
\hline-291.5 & 0.0164 & $\mid 8^{\wedge} 1>$ \\
\hline-356.1 & 0.0066 & $\left|3^{\wedge} 3\right\rangle$ \\
\hline-406.1 & 0.0031 & $\mid 11^{\wedge} 1>$ \\
\hline-410.2 & 0.0196 & $\mid 8^{\wedge} 1 ; 3^{\wedge} 1>$ \\
\hline-524.8 & 0.0025 & $\mid 11^{\wedge} 1 ; 3^{\wedge} 1>$ \\
\hline-528.9 & 0.0123 & $\mid 8^{\wedge} 1 ; 3^{\wedge} 2>$ \\
\hline-555.0 & 0.0027 & $\mid 16^{\wedge} 1>$ \\
\hline-583.0 & 0.0075 & $\mid 8^{\wedge} 2>$ \\
\hline-603.3 & 0.0039 & $\mid 19^{\wedge} 1>$ \\
\hline-647.6 & 0.0053 & $\mid 8^{\wedge} 1 ; 3^{\wedge} 3>$ \\
\hline-673.6 & 0.0029 & $\mid 16^{\wedge} 1 ; 3^{\wedge} 1>$ \\
\hline-701.7 & 0.0095 & $\mid 8^{\wedge} 2 ; 3^{\wedge} 1>$ \\
\hline-722.0 & 0.0036 & $\mid 19^{\wedge} 1 ; 3^{\wedge} 1>$ \\
\hline-820.4 & 0.0063 & $\mid 8^{\wedge} 2 ; 3^{\wedge} 2>$ \\
\hline-854.1 & 0.0142 & $\left|30^{\wedge} 1\right\rangle$ \\
\hline-866.7 & 0.0029 & $\left|32^{\wedge} 1\right\rangle$ \\
\hline-874.5 & 0.0028 & $\mid 8^{\wedge} 3>$ \\
\hline-939.1 & 0.0029 & $\left|8^{\wedge} 2 ; 3^{\wedge} 3\right\rangle$ \\
\hline-972.8 & 0.0155 & $\left|30^{\wedge} 1 ; 3^{\wedge} 1\right\rangle$ \\
\hline-985.4 & 0.0031 & $\left|32^{\wedge} 1 ; 3^{\wedge} 1\right\rangle$ \\
\hline-993.1 & 0.0038 & $\mid 8^{\wedge} 3 ; 3^{\wedge} 1>$ \\
\hline-1091.5 & 0.0089 & $\mid 30^{\wedge} 1 ; 3^{\wedge} 2>$ \\
\hline-1111.8 & 0.0026 & $\mid 8^{\wedge} 3 ; 3^{\wedge} 2>$ \\
\hline-1145.6 & 0.0082 & $\left|30^{\wedge} 1 ; 8^{\wedge} 1\right\rangle$ \\
\hline-1210.2 & 0.0036 & $\mid 30^{\wedge} 1 ; 3^{\wedge} 3>$ \\
\hline-1264.3 & 0.0097 & $\mid 30^{\wedge} 1 ; 8^{\wedge} 1 ; 3^{\wedge} 1>$ \\
\hline-1383.0 & 0.0061 & $\mid 30^{\wedge} 1 ; 8^{\wedge} 1 ; 3^{\wedge} 2>$ \\
\hline-1437.1 & 0.0035 & $\mid 30^{\wedge} 1 ; 8^{\wedge} 2>$ \\
\hline-1501.7 & 0.0026 & $\left|30^{\wedge} 1 ; 8^{\wedge} 1 ; 3^{\wedge} 3\right\rangle$ \\
\hline-1555.8 & 0.0044 & $\left|30^{\wedge} 1 ; 8^{\wedge} 2 ; 3^{\wedge} 1\right\rangle$ \\
\hline-1674.5 & 0.0029 & $\mid 30^{\wedge} 1 ; 8^{\wedge} 2 ; 3^{\wedge} 2>$ \\
\hline-1708.3 & 0.0051 & $\mid 30^{\wedge} 2>$ \\
\hline-1827.0 & 0.0055 & $\left|30^{\wedge} 2 ; 3^{\wedge} 1\right\rangle$ \\
\hline-1945.7 & 0.0031 & $\mid 30^{\wedge} 2 ; 3^{\wedge} 2>$ \\
\hline-1999.8 & 0.0027 & $\mid 30^{\wedge} 2 ; 8^{\wedge} 1>$ \\
\hline-2118.5 & 0.0032 & $\mid 30^{\wedge} 2 ; 8^{\wedge} 1 ; 3^{\wedge} 1>$ \\
\hline
\end{tabular}

Table S1-A. List of most intense vibronic bands in calculated spectra of 1,4-DAT. For description, see Table S1. 

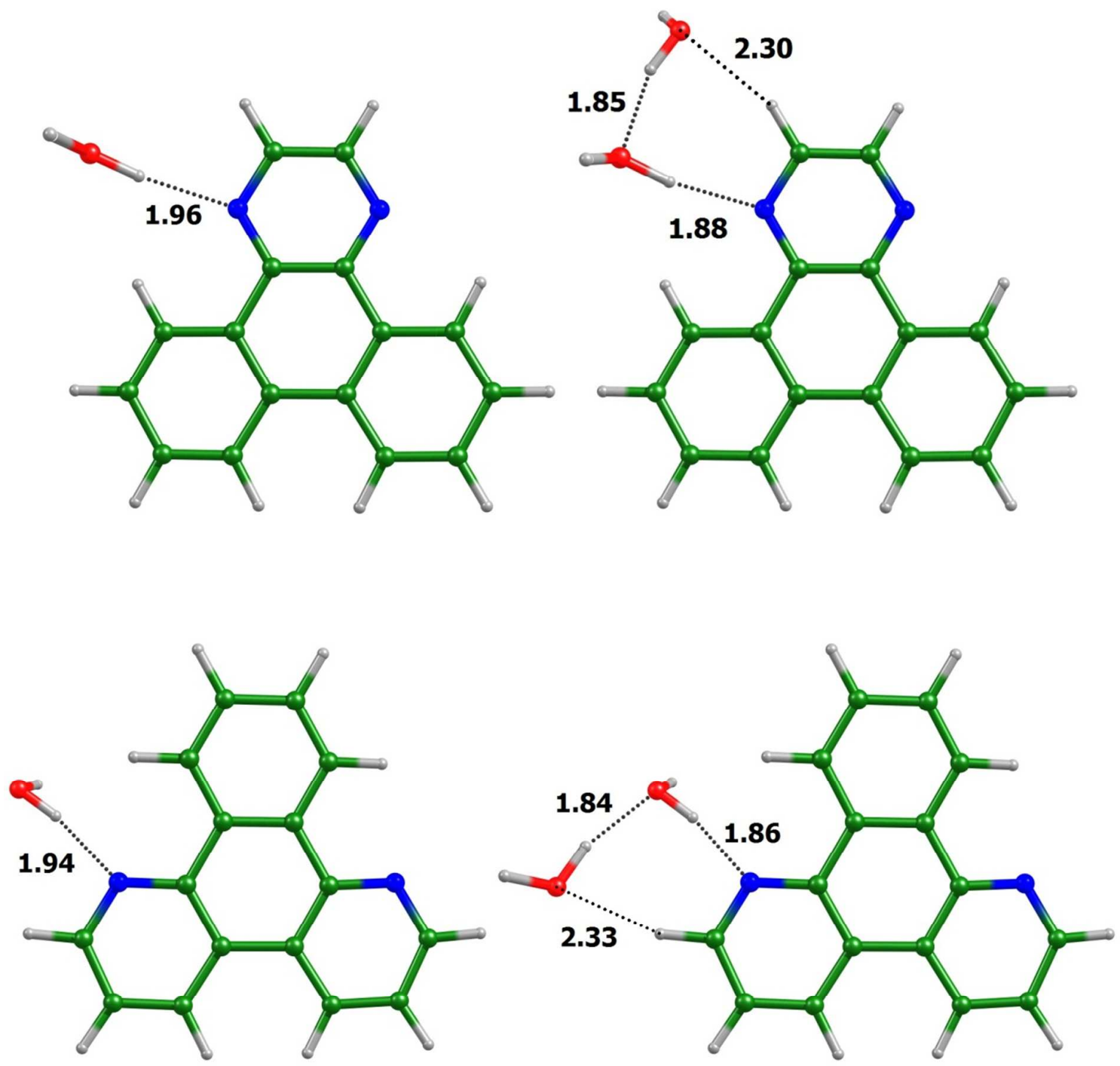

Figure S5. Ground state geometries of 1:1 and 1:2 complexes of 1,4-DAT (up) and 1,8-DAT (bottom) with water calculated at the DFT/B3LYP level. Hydrogen-bond distances are given in angstroms. 